\title{
Does LIN28B gene dysregulation make women more likely to abort?
}

\author{
QiaoYao Huang'1, YanRu Niu², LiJun Song1', JinZhi Huang1', Chenxi Wang1 and TianZhong Ma®1 \\ 1Reproductive Medicine Center, Affiliated Hospital of Guangdong Medical University, Zhanjiang, Guangdong, China \\ 2Laboratory of Minimally Invasive Orthopaedics. Guangdong Medical University, Zhanjiang, Guangdong, China
}

Correspondence should be addressed to T Ma: tiann8283@163.com

\begin{abstract}
Background: LIN28B plays an important role in early embryonic development, but its role in villous trophoblast implantation and differentiation remains unknown. This study aims to verify the role of LIN28B in trophoblastic villous tissue and cells from women with URSA (unexplained recurrent spontaneous abortion) and artificial termination of pregnancy (negative control, NC).

Methods: The LIN28B gene and its protein expression level were detected with real-time quantitative PCR, Western immunoblotting analysis, and immunocytochemistry. The gene was also overexpressed in chorionic villous cell lines (HTR-8/SVneo and BeWo) to examine its effect on trophoblast function.

Results: The expression of LIN28B mRNA and protein of URSA villi was lower than that in the NC group. At the cellular level, overexpression of LIN28B enhanced cellular migration, and invasion, and inhibited apoptosis. LIN28B may inhibit apoptosis by promoting Akt phosphorylation and by inhibiting Bad phosphorylation and Bcl-2 expression. In addition, LIN28B inhibited cell fusion and reduced cellular syncytia.

Conclusions: LIN28B can inhibit cell invasion and migration in vitro and promote apoptosis and fusion. The low expression of LIN28B in URSA villous trophoblast cells may be one of the causes of abortion. The role of LIN28B in villous trophoblasts needs further study.
\end{abstract}

\section{Lay summary}

Propagation of offspring is of great significance to the continuation of the human race. However, continuous pregnancy is more difficult for some women, especially women who have multiple miscarriages. One important contributor is the cessation of development caused by genetic factors of the embryo, but there are still many unknown reasons. We investigated the LIN28B gene which is a possible pathogenic factor in the placenta. We collected 25 cases of abortion in the experimental group (unexplained recurrent abortion group) and 25 in the control group (artificial termination of pregnancy group): on average at 7-8 weeks of pregnancy. We tested the function of lin28b in these samples and verified its function in cell lines. LIN28B plays an important role in maintaining early pregnancy by promoting the invasion of villous cells, inhibiting apoptosis and fusion, and the reduction of LIN28B expression may lead to the occurrence of early miscarriage.

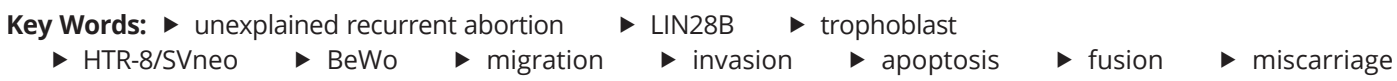

Reproduction and Fertility (2021) 2 211-220

https://raf.bioscientifica.com

https://doi.org/10.1530/RAF-21-0033
(C) 2021 The authors Published by Bioscientifica Ltd

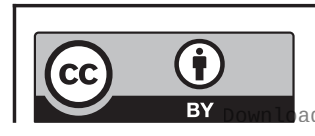

This work is licensed under a Creative Commons Attribution 4.0 International License. 


\section{Background}

Recurrent spontaneous abortion (RSA 2012) refers to the failure of two or more pregnancies. Its etiology is complex and lacks specific clinical manifestations, with genetic factors comprising the main causes of early abortion (Ogasawara et al. 2000, Stephenson et al. 2002). However, with $50 \%$ of RSA still occurring due to unknown causes, we designate this as unexplained recurrent abortion (URSA) (RSA 2012). URSA is an excluded diagnostic disease, and its diagnosis and treatment still face great challenges, although studies have shown that the underlying causes of RSA include systemic endocrine and immune disorders (Mekinian et al. 2012, RSA 2012, Coomarasamy et al. 2015, Motedayyen et al. 2018, Canfield et al. 2019, Xu et al. 2019). Many scholars are now paying close attention to the gamete and the regulation of maternal-fetal interactions after blastocyst implantation (Bao et al. 2013, Knöfler \& Pollheimer 2013, Xu et al. 2017). Gestation is a complex and delicate process and the maintenance of pregnancy depends upon the normal functioning of trophoblast cells. The fertilized oocyte subsequently develops into a blastocyst, with implantation usually occurring 6-7 days after fertilization. The trophectoderm (with a high differentiation potential) and the maternal endometrial epithelial cells undergo a series of complex interactions that allows embryonic contact, adherence, and implantation into the endometrium to ensure successful implantation. During this period, the primitive syncytium is generated by the fusion of early cytotrophoblastic cells. As the placental villi continue to develop, primary villi composed of cytotrophoblast and syncytiotrophoblast are formed (Zhou et al. 1997, Gamage et al. 2016). Normal functions, such as differentiation, migration, and invasion of chorionic trophoblast cells play an important role in the maintenance of pregnancy. A series of pregnancy-related diseases (including miscarriage and preeclampsia) may then be caused by the biologic dysfunction of trophoblast cells (insufficient differentiation, excessive apoptosis, impaired proliferation, or decreased invasiveness) (Huang et al. 2014, Windsperger et al. 2017, Wu et al. 2017, Yang et al. 2018, Canfield et al. 2019).

LIN28 was first found to be encoded by the Lin28b gene on chromosome 6q21 when the sequence gene was screened within C. elegans (Ambros and Horvitz 1984), and its expression is histologically specific, localized primarily in the placenta, testis, and fetal liver. There are two congeners of LIN28A and LIN28B in mammals: these are highly conserved RNA-binding proteins, and LIN28B is a highly conserved structural protein in advanced eukaryotes
(Thornton \& Gregory 2012, Shyh-Chang \& Daley 2013). Although LIN28B RNA-binding proteins play an important role in embryonic development and implantation (Lozoya et al. 2014, Zhao et al. 2018), the mechanism of action in the chorionic trophoblast of URSA remains unclear. We herein explore a possible role for LIN28B in placental trophoblast in URSA patients at the tissue and cellular levels.

\section{Methods}

\section{Experimental object and specimen collection}

\section{Experimental object}

The protocol governing the subjects of this study was approved by the Ethics Committee of the Affiliated Hospital of Guangdong Medical University (PJ2013014). Patients with unexplained recurrent abortion (URSA group) or unexpected pregnancy which refers to accidental pregnancy caused by the failure of contraceptive measures (usually refers to a married woman who has an unplanned pregnancy) (<12 weeks) (NC group) were selected from the Outpatient Department of the Affiliated Hospital of Guangdong Medical University from 2016 to 2018. Each group entailed 25 cases of chorionic villi. The mean age of the URSA group was $33.73 \pm 5.33$ years (mean \pm S.D.), and the mean gestational age was $7.82 \pm 0.81$ weeks. The mean age of the NC group was $31.23 \pm 4.83$, and the mean gestational age was $7.31 \pm 0.95$ weeks. All of the patients signed informed consent forms. The inclusion criteria for the URSA group were all of the patients who underwent two or more miscarriages, possessed excluded possible causes (chromosomal abnormalities, immunological factors, etc.), and were diagnosed as having early abortion villi of URSA ( $<12$ weeks). For the NC group, chronologic and gestational age were similar, and the patients who voluntarily requested termination of pregnancy had a history of normal pregnancy and delivery before the current pregnancy, had no stillbirths, no history of spontaneous abortion, no medication use, and no history of viral infection. During the current pregnancy, there were no threatened abortion symptoms or signs, and B-ultrasonography suggested normal embryonic development. There was no statistical difference in age and gestational age between the two groups $(P>0.05)$.

\section{Specimen collection}

The villi were extracted immediately in the aseptic state, washed with sterile saline until no obvious blood was observed. The villi were divided into three parts (some

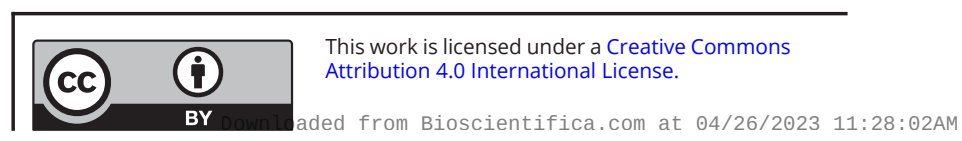


of the villi were quickly placed into cryopreserved tubes containing $1 \mathrm{~mL}$ of Trizol reagent (Invitrogen)). Tissues were placed into a cryotube, quickly deposited into liquid nitrogen for quick freezing, and stored in a $-80^{\circ} \mathrm{C}$ refrigerator (Invitrogen) for later extraction of tissue protein and RNA. Partial fixation in $4 \%(\mathrm{v} / \mathrm{v})$ formaldehyde was used for immunohistochemistry.

\section{Cell culture}

HTR-8/SVneo or BeWo cells from the Stem Cell R\&D and Clinical Transformation Center of the Affiliated Hospital of Guangdong Medical University were cultured in RPMI 1640 medium (Gibco) containing 10\% (v/v) fetal bovine serum (FBS, Gibco) and placed in an incubator with a $\mathrm{CO}_{2}$ concentration of $5 \%$, a humidity of $95 \%$, and a temperature of $37^{\circ} \mathrm{C}$. Different cell lines were developed to study trophoblast functions (cell fusion, migration, and invasion) including BeWo and HTR-8/SVneo. Studies have shown that BeWo is positive for the trophoblast/epithelial marker CK7, while HTR-8/SVneo cells contain almost no CK7 positive cell clusters. Studies have shown that BeWo is more used to study cell fusion, while HTR-8/SVneo is inclined to invasion and migration functions.

\section{Transfection}

For HTR-8/SVneo or BeWo cells, Lipofectamine 3000 (Invitrogen) was used to transfect plasmids (a LIN28B overexpression plasmid was constructed with green fluorescent GFP, EX-Y3355-Lv105,GeneCopoeia, US). Transfection was carried out according to the specifications of the transfection reagent. Transfection efficiency was about $70 \%$.

\section{Immunohistochemistry}

Immunohistochemical staining using a primary antibody (LIN28B, Abcam) was performed on sections of placental villi. According to the instructions for the SP Rabbit and
Mouse HRP Kit (DAB) (CWBIO, China), biotinylated antirabbit/mouse universal second antibody was combined with the first specific antibody, and the second antibody was labeled with biotin combined with the streptavidinlabeled peroxidase (HRP). A streptavidin complex was thereby achieved by labeling with antigen-specific first antibody-biotinylated second antibody-HRP. Blank control: the primary antibody was replaced with PBS, other steps were as previously mentioned (including endogenous peroxidase blocking and serum blocking). We used ImageJ software to carry out a sample image for immunohistochemistry and made quantitative comparisons using digital image analysis.

\section{RNA and protein extraction}

We used collected tissue or untreated cell samples to which Trizol (Invitrogen) was added and ground and homogenized to extract RNA, while the protein was extracted by homogeneous lysis of RIPA lysate (Beyotime, China) containing 1\% PMSF (Beyotime, China).

\section{Real-time quantitative PCR (qPCR)}

cDNA was synthesized according to the manufacturer's instructions found in the PrimeScriptRT Reagent Kit using gDNA Eraser (TaKaRa, Japan) kit, and the TB Green chimeric fluorescence method (TaKaRa, Japan) was used for real-time quantitative PCR. An ABI7500 real-time qPCR system was used to perform all qPCR reactions, and the data were standardized to $\beta$-actin. The qPCR primer sequence is shown in Table 1 . We analyzed relative gene expression using the semi-quantitative $2^{-\Delta \Delta C t}$ method.

\section{Western blotting analysis}

As mentioned previously, total protein lysates were separated by PAGE, transferred to PVDF membranes (Millipore), and sealed at room temperature with $5 \%(\mathrm{w} / \mathrm{v})$ milk (BD, US) for $2 \mathrm{~h}$. Lysates were incubated with LIN28B

Table 1 Primers used for qPCR experiments.

\begin{tabular}{|c|c|c|c|}
\hline \multirow[b]{2}{*}{ Gene } & \multirow[b]{2}{*}{ NCBI accession no. } & \multicolumn{2}{|c|}{ Primer sequence $\left(5^{\prime}-3^{\prime}\right)$} \\
\hline & & Forward & Reverse \\
\hline LIN28B & NM_001004317 & CCAGCCATGCACTTCAACTCTCC & TGACCTGCCTGACCGTTCTGAG \\
\hline$A C T B$ & NM_001101 & GGCACCACACCTTCTACAATGAGC & GATAGCACAGCCTGGATAGCAACG \\
\hline $\mathrm{CDH1}$ & NM_004360 & GCTCTTCCAGGAACCTCTGTGATG & AAGCGATGGCGGCATTGTAGG \\
\hline $\mathrm{CDH} 3$ & NM_001793 & GAGAACCTGAAGGCGGCTAACAC & CTTGGTCGGAGGCGGAGGAG \\
\hline VIM & NM_003380 & TTGCCGTTGAAGCTGCTAACTACC & AATCCTGCTCTCCTCGCCTTCC \\
\hline $\mathrm{CDH} 2$ & NM_001792 & AGGCGTCTGTAGAGGCTTCTGG & GAGGCTGTCCTTCATGCACATCC \\
\hline$E R V W-1$ & NM_001130925.1 & CCCCATCGTATAGGAGTCTTTC & GTTTGGGTGAAGTAAGTCCAAC \\
\hline https://r & $\begin{array}{l}\text { scientifica.com } \\
\text { 5/10.1530/RAF-21-0033 }\end{array}$ & $\begin{array}{r}\text { (c) } 2021 \text { The authors } \\
\text { Published by Bioscientifica Ltd }\end{array}$ & $\begin{array}{l}\text { This work is licensed under a Creative Commons } \\
\text { Attribution } 4.0 \text { International License. }\end{array}$ \\
\hline
\end{tabular}


(Abcam), $\beta$-actin, Akt, p-Akt, Bad, p-Bad, or Bcl-2 primary antibodies overnight at $4^{\circ} \mathrm{C}$ and then incubated with secondary antibody (CST,US) for $1 \mathrm{~h}$ at room temperature. The resulting immunoblot was scanned and quantitatively assessed using a Tanon 5200 imaging system.

\section{Invasion and migration assays}

HTR-8/SVneo or BeWo cells were seeded in a 6-well culture dish and transfected with a plasmid on the next day. After $24 \mathrm{~h}$, the transfected cells were digested and resuspended in serum-free RPMI 1640 medium and inoculated to an upper chamber (Corning) that had been coated with matrigel (Corning); the lower chamber was placed in 10\% $(v / v)$ FBS. After 1 or 2 days, the non-invasive cells in the upper chamber were wiped with a swab, and the remaining invasive cells were then stained with crystal violet and imaged. We counted five fields of view in each cell, and the numbers of invasive cells were analyzed statistically. The steps with respect to migration were the same as for invasion, but the inoculated upper chamber did not need to be covered with matrigel.

\section{Apoptosis assay}

FACSCantoII flow cytometry (BD, US) was used to measure apoptosis according to the instructions in the PE Annexin $\mathrm{V}$ apoptosis kit maker (BD, US). Cells that are considered viable are PE Annexin V and 7-AAD negative; cells that are in early apoptosis are PE Annexin $\mathrm{V}$ positive and 7-AAD negative; cells that are in late apoptosis or already dead are both PE Annexin V and 7-AAD positive.

\section{Evaluation of cell cycle kinetics}

According to the manufacturer's instructions found in the cell cycle and apoptosis detection kit (Beyotime, China), we fixed cells with $70 \%(\mathrm{v} / \mathrm{v})$ ethanol for $4 \mathrm{~h}$ at $4^{\circ} \mathrm{C}$ and then added pyridine iodide staining solution (for a 0.5 $\mathrm{mL}$ sample we used $25 \mu \mathrm{L}$ of $20 \times$ staining buffer and 10 $\mu \mathrm{L}$ of $50 \times$ RNase $\mathrm{A}$ at $37^{\circ} \mathrm{C}$ for $30 \mathrm{~min}$ ) and measured cell cycle parameters by FACSCantoII flow cytometry (BD, US) within $24 \mathrm{~h}$.

\section{Cell fusion assay}

BeWo cells were inoculated in confocal dishes and transfected with plasmid when the cell density was 30-50\%. After $12 \mathrm{~h}, 50 \mu \mathrm{M}$ adenylate cyclase activator (forskolin, FSK, Beyotime, China) and 0.1\% (v/v) DMSO were added consecutively. After continuous culture for 1 or 2 weeks, we immobilized the cells with $4 \%(\mathrm{v} / \mathrm{v})$ paraformaldehyde, incubated them overnight with $\beta$-catenin (Abcam) at $4^{\circ} \mathrm{C}$, and then incubated them additionally with secondary antibody (CST) for $1 \mathrm{~h}$ at room temperature. After staining cells with DAPI, we used a laser confocal microscope (Olympus FV3000) to photograph and analyze results. The full field of view of the confocal dish was taken using a $20 \times$ objective, and the number of cells with $\geq 3$ nuclear fusions was counted.

\section{Statistical analysis}

The results are expressed as mean \pm S.E. The normality and variance equivalence of all data were tested to determine the appropriate statistical test. We used Student's $t$-test, $x^{2}$, or Fisher's exact probability test to determine significant differences between groups. All statistical analyses were processed using GraphPad Prism 7 software and a difference of $P<0.05$ was considered statistically significant, except where noted. The number of experimental replicates and the number of replicates within each experiment were both three times.

\section{Results}

\section{The URSA group showed decreased expression of} LIN28B mRNA and protein

We verified the expression of LIN28B in URSA villi by IHC, WB, and qPCR. The results of IHC showed that the expression of LIN28B in the chorionic villi of the URSA group $(6.76 \pm 0.87)$ was lower than that of the NC group $(12.75 \pm 0.57)(P=0.0012)$ and that LIN28B was expressed in the nucleus and cytoplasm of the villous epithelial tissue but not in the villous interstitial tissue (Fig. 1A). qPCR results showed that the LIN28B mRNA expression level was lower in the URSA group $(0.53 \pm 0.16)$ relative to the NC group $(1.18 \pm 0.21)(P=0.003)$ (Fig. 1B). The WB results also showed that LIN28B protein in the URSA group $(0.53 \pm 0.07)$ was lower than that in the NC group $(0.93 \pm 0.07)(P=0.007)$ (Fig. 1C).

\section{LIN28B can be overexpressed in HTR-8/SVneo and BeWo cells}

To verify the possible role of LIN28B in placental villous trophoblastic cells from women with URSA, we selected two common cell lines that represent placental function (HTR-8/SVneo is primarily used to detect invasiveness

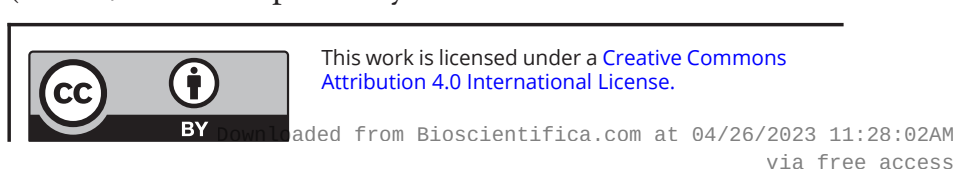


A

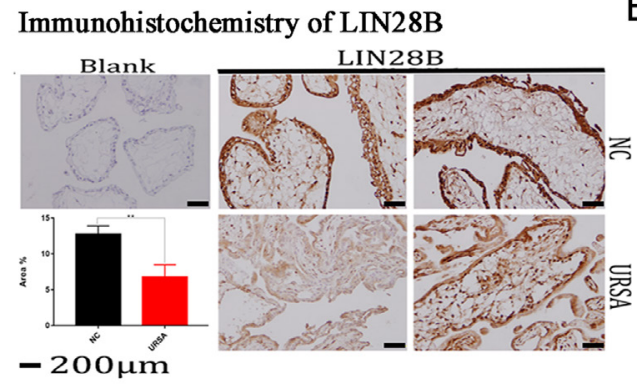

C LIN28B protein expression

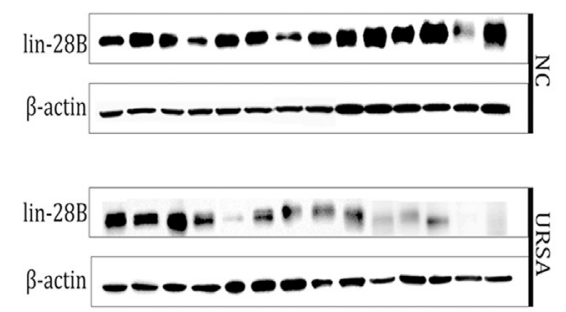

B LIN28B mRNA expression

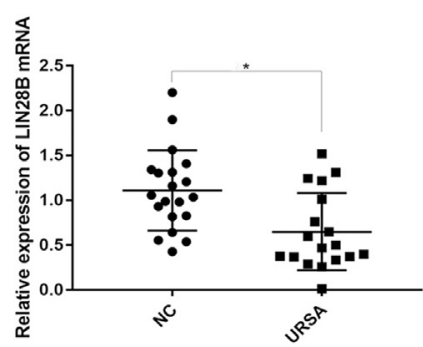

Figure 1 Low expression of LIN28B in placental villi of unexplained recurrent abortion (URSA). (A) Immunohistochemical analysis of LIN28B in NC villus trophoblast (middle and upper right) compared with URSA tissue samples (middle and lower right) and blank control (left), quantitative analysis of two groups (bottom left) $(n=25)$. Blank control: the primary antibody was replaced with PBS. (B) The relative expression of LIN28B mRNA in placental villi of URSA and NC $(n=20)$. (C) The expression of LIN28B protein in URSA and NC villi $(n=14)$. The number of experimental

replicates and the number of replicates within each experiment were both three times. $\star \star \star * P<0.0001, * \star \star P P 0.001, * \star P<0.01, * P<0.05$. and BeWo for fusion induction). Based on the low LIN28B expression of HTR-8/SVneo and BeWo, we constructed a LIN28B overexpression plasmid (LIN28B-o) and the corresponding empty vector (vector). One day (D1), 2 days (D2), and 3 days (D3) after transfection, we used fluorescence microscopy to quantify plasmid fluorescence (Fig. 2A), and the transfection effect was verified by qPCR (Fig. 2B) and Western immunoblotting analysis (Fig. 2C). Results showed that the expression of LIN28B in the overexpression group was significantly increased compared with the control group and that the cell model was successfully constructed.

\section{LIN28B promotes cellular migration and invasion}

We found that invasion (Fig. 2E) and migration (Fig. 2F) of the LIN28B overexpression group increased in both HTR-8/SVneo and BeWo cells, but there was no significant difference in the proliferative capacity between the two groups (Fig. 2D). Cell cycle detection (Fig. 2D) showed that compared with the vector group, the proportion of cells entering $S$ phase increased (HTR-8/SVneo, $37.52 \pm 2.04$ (D1), $37.5 \pm 1.89$ (D2), $40.97 \pm 0.54$ (D3); BeWo, $33.17 \pm 2.13$ (D1), $30.37 \pm 1.58$ (D2), $32.16 \pm 0.75$ (D3) vs LIN28-o group: HTR-8/SVneo, $43.78 \pm 4.13$ (D1), $46.66 \pm 0.94$ (D2), $42.25 \pm 0.31$ (D3); BeWo, $36.15 \pm 3.71$ (D1), $28.71 \pm 1.70$ (D2), $39.8 \pm 0.54$ (D3)) $(P>0.05)$. No significant difference was observed in cell proliferation. The invasive ability of HTR-8/SVneo or BeWo cells in the LIN28B-o group was two to three times that of the control group (Fig. 2E), and the migratory capability was three times higher than that in the control group $(P<0.05)$ (Fig. 2E and F).

\section{LIN28B inhibits cellular apoptosis}

Flow cytometry showed that the percentages of cells in early, late, and total apoptosis in the LIN28-o group were lower than in the vector group (Fig. 3A). In HTR-8/SVneo cells, only the difference on D1 was statistically significant $(P=0.002)$. In BeWo cells, the apoptotic index on D1 remained unchanged $(P>0.05)$, but the apoptosis on D2 $(P=0.03)$ and D3 $(P=0.003)$ significantly decreased. To further prove that LIN28B inhibits apoptosis, we confirmed protein expression for Akt/Bad/Bcl-2 and their phosphorylated states (Fig. 3B) and found that p-Akt in HTR-8/SVneo cells in the LIN28-o group increased $(P>0.05)$ and in p-Bad $(P<0.05)$ and Bcl-2 decreased $(P>0.05)$. In the LIN28-o group, only p-Akt increased in BeWo cells $(P<0.05)$.

\section{LIN28B inhibits cellular fusion}

Our study found that BeWo cells in the LIN28B-o group were more likely to inhibit cell fusion than cells in the vector group. After induction with $50 \mu \mathrm{M}$ FSK, we observed with laser confocal microscopy that the number of cells fused at 1 or 2 weeks in the vector group was higher than that in the LIN28B-o group (Fig. 4A and B). To further verify that LIN28B was associated with cell fusion, we used qPCR to detect the expression of ERVW-1 (a factor known to be involved in cell fusion) and also evaluated

\section{This work is licensed under a Creative Commons} Attribution 4.0 International License. 
A GFP fluorescence

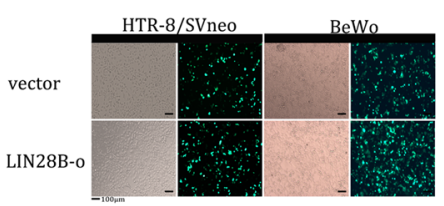

C

Lin-28B protein expression in cell after transfection

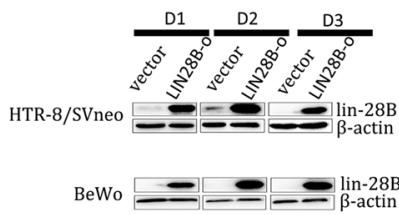

E

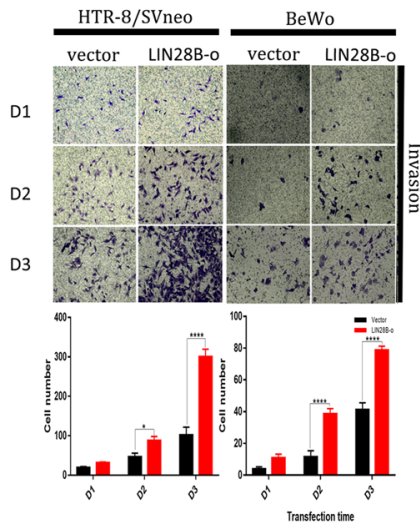

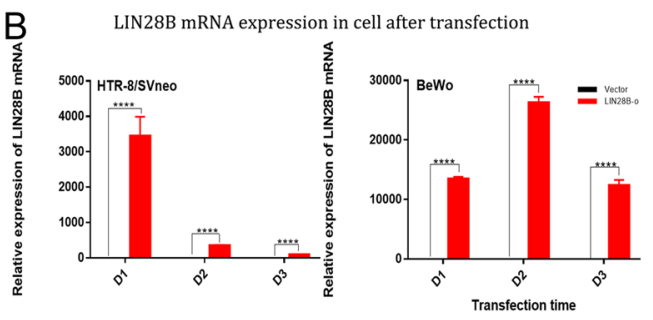

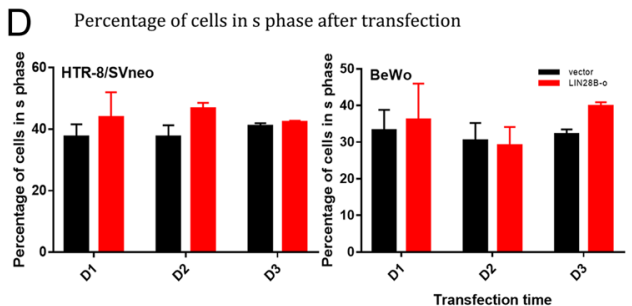

$\mathrm{F}$

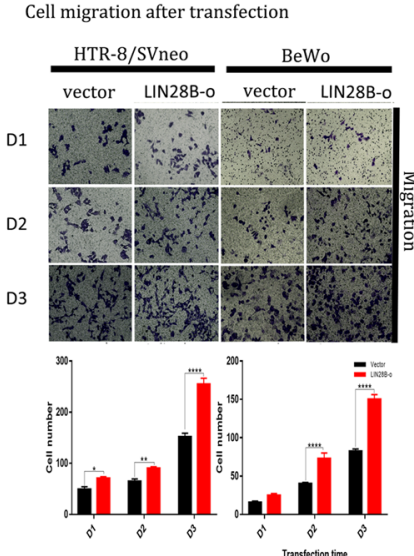

Figure 2 LIN28B promotes invasion and migration of HTR-8/SVneo and BeWo cells. (A) Transfection of HTR-8/SVneo and BeWo cells into empty vector control group (vector) and LIN28B overexpression plasmids (LIN28B-o) with fluorescence photos. (B and C) qPCR and Western blot analysis of vector and LIN28B-o groups after transfection of HTR-8/SVneo and BeWo cells into empty vector control group were performed on 1 day (D1), 2 days (D2), 3 days (D3). (D) The percentage of HTR-8/SVneo and BeWo cells transfected with vector and LIN28B-o plasmid D1, D2, D3 into S phase by flow cytometry. (E and F) The representative microphotographs of invasive cells in transwell invasion assay (plus Matrigel) and migration cells in transwell migration assay. The number of experimental replicates and the number of replicates within each experiment were both three times. ${ }^{*} * \star p<0.0001$ $\star * * P<0.001, * * P<0.01, * P<0.05$
E-cadherin (CDH1), P-cadherin (CDH3), vimentin (VIM), and N-cadherin (CDH2), factors related to epithelialinterstitial transformation. Our results showed that the expression of ERVW-1 in the vector group was higher than in the LIN28B-o group $(P=0.005)$ and that the expression of the epithelial-related factors $\mathrm{CDH} 1$ and $\mathrm{CDH} 3$ was lower than in the LIN28B-o group after induction with $50 \mu \mathrm{M}$ FSK for 1 or 2 weeks $(P<0.05)$. VIM expression in the vector group after 1 week of induction was higher than that of the LIN28B-o group $(P<0.05)$, while the remaining indices showed a decreasing tendency $(P<0.05)$ (Fig. 4C).

\section{Discussion}

In the present study, we have for the first time detected the expression of LIN28B in villi from women with URSA and have explored a possible role for LIN28B in URSA. LIN28B is a paternal imprinting gene (Davis et al. 2015) (i.e. expressed by the paternal source) that promotes the development of the placenta and also stimulates the embryo or fetus to extract nutrients from the mother, thereby promoting the growth of the individual (Moore 2001, Huppertz 2008, Monk 2015). Our study found that the expression of LIN28B in the villous tissue of URSA patients decreased, implying that the expression of the parental imprinting gene LIN28B may inhibit the development of trophoblast cells and lead to the occurrence of miscarriage.

Recentstudies have shown that changes in trophoblastic function are realized through various signal transduction pathways (Chan etal. 2013, Seabrook et al. 2013, Lozoya et al. 2014, Lin et al. 2018, Canfield et al. 2019). LIN28 is highly expressed in the placenta and plays an important role in embryonic development and implantation as an RNAbinding protein (Canfield et al. 2019). Some studies have shown that the increase in LIN28B expression is positively correlated with the invasion, migration, and proliferation of trophoblast cells (Thornton \& Gregory 2012, Shyh-Chang \& Daley 2013, Park et al. 2017, West et al. 2019). Canfield et al. (2019) found that invasive interstitial EVT expressed higher levels of LIN28B in placental sections during early pregnancy compared with non-invasive proximal trophoblast cells,

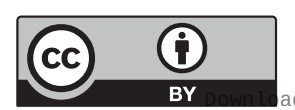


A
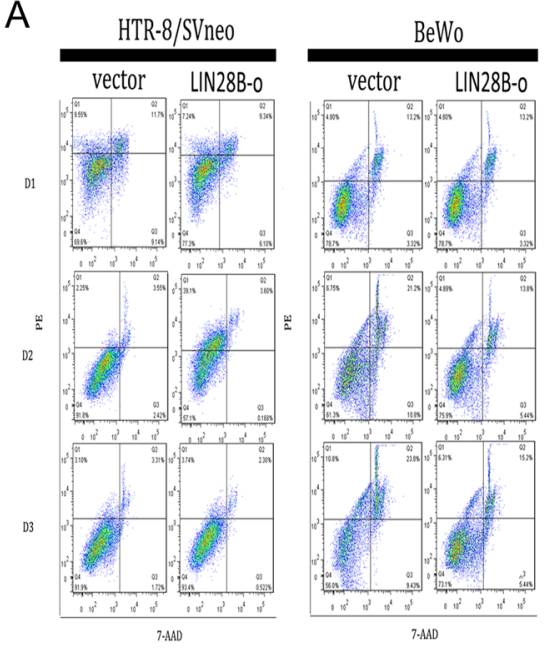

B

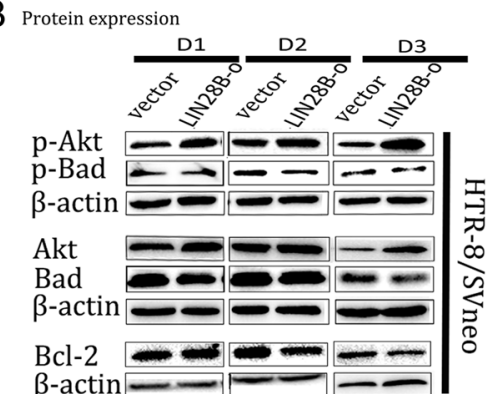

Bcl-2

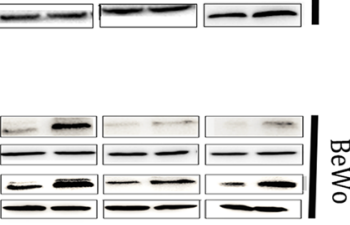

HTR-8/SVneo
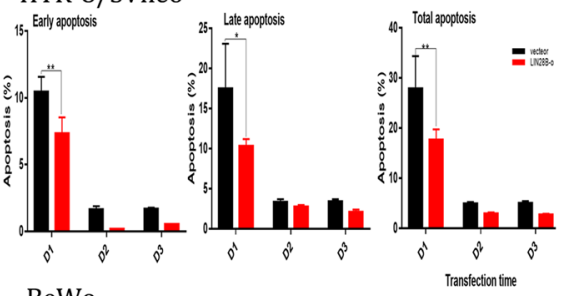

BeWo
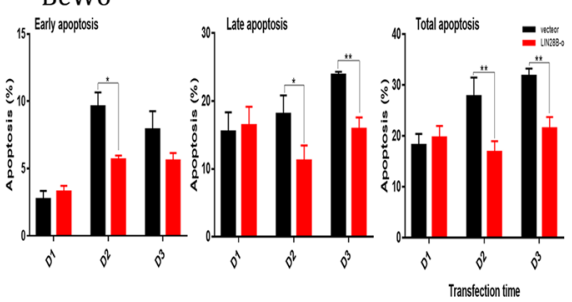

HTR-8/SVneo
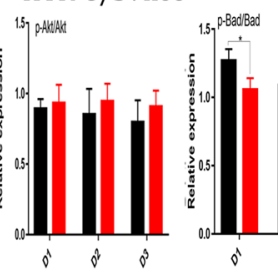

.
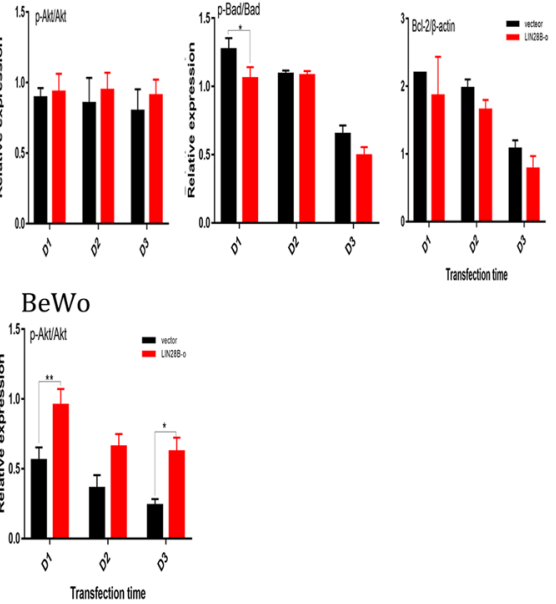

Figure 3 LIN28B inhibits apoptosis of HTR-8/ SVneo and BeWo cells. (vector) and LIN28B overexpression (LIN28B-o) plasmids were transfected into empty vector control group after 1 day (D1), 2 days (D2), 3 days (D3). (A) Flow cytometry was used to determine the apoptosis of HTR-8/SVneo and BeWo cells by flow cytometry (FCM) staining with annexin $\mathrm{V}$ and

7-aminoactinomycin (7-AAD). The results showed that the percentage of apoptotic cells was early, late-stage, and total apoptotic cells (annexin V positive). (B) The expression of total protein and phosphorylated protein of Akt/Bad/Bcl-2 apoptosis-related pathway in HTR-8/SVneo and BeWo cells transfected with vector and LIN28B-o plasmids D1/D2/D3 was studied. The number of experimental replicates and the number of replicates within each experiment were both three times. $* \star \star \star x P<0.0001, * \star * P<0.001$, $\star * P<0.01, * P<0.05$.

and an increased expression of LIN28B increased HTR8/ SVneo cell proliferation, migration, and invasion in vitro. We demonstrated that HTR-8/SVneo and BeWo cells that overexpress LIN28B possessed enhanced migratory, and invasive capabilities, consistent with previous studies. Normal pregnancy requires adequate early EVT to invade the decidua, a situation highly similar to cancer metastasis with the proper remodeling of the spiral artery (Velicky et al. 2016, Chang et al. 2018, Pollheimer et al. 2018). Lin et al. (2018) found that LIN28B may inhibit apoptosis of ovarian cancer cells through the AKT2/FOXO3A/BIM axis. To further investigate the possible role of LIN28B in trophoblast cells, we also examined the apoptotic function and apoptosis-related proteins of HTR-8/SVneo and BeWo cells that overexpress LIN28B and discovered that antiapoptotic ability was enhanced after overexpression of LIN28B, that the phosphorylation of the anti-apoptotic protein Akt increased, and that the phosphorylation of the pro-apoptotic proteins Bad and Bcl-2 decreased in
HTR-8/SVneo cells. In BeWo cells, we only detected an increase in phosphorylation of the anti-apoptotic protein Akt, which may have been due to either extremely low or very unstable protein phosphorylation in BeWo cells after overexpression of LIN28B. We hypothesized that LIN28B inhibited apoptosis of villous cells through the Akt/Bad/Bcl-2 signaling pathway, thereby changing the migration and invasion of villi and ensuring the smooth progress of the pregnancy. The low expression of LIN28B in villous tissue of URSA patients may accelerate dysfunction and apoptosis in trophoblast cells, leading to the occurrence of abortion. But there were limitations of this experiment. Bad, Bcl2, and Akt not only affect the mechanism of apoptosis but also affect many other cell activities. More apoptosis evaluation experiments should be added, such as: adding apoptosis inhibitor (zVad-fmk), detecting apoptosisrelated factors such as caspase-3 and PARP.

Cell fusion in mammals is a common physiologic process that is involved in fertilization, placental

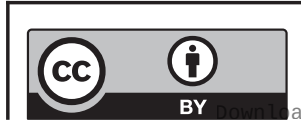

This work is licensed under a Creative Commons Attribution 4.0 International License. 
A
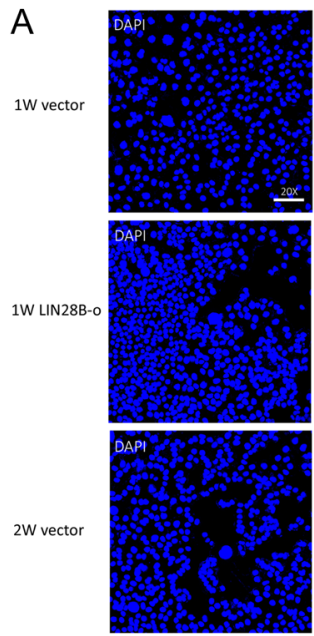

2W LIN28B-0
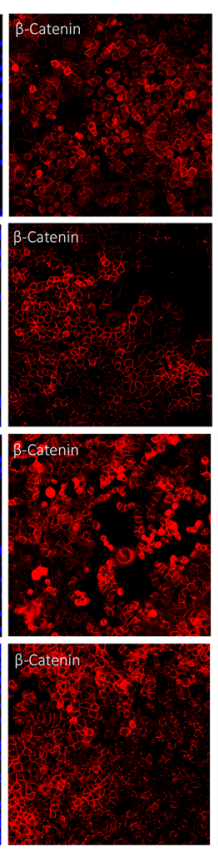
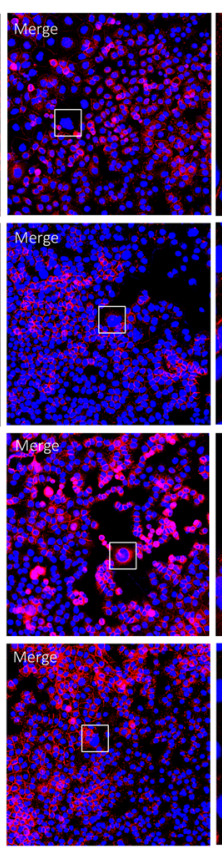
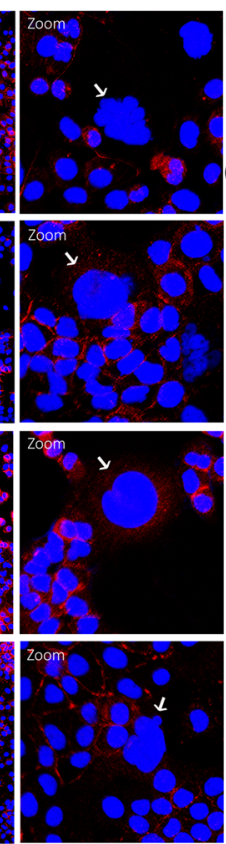

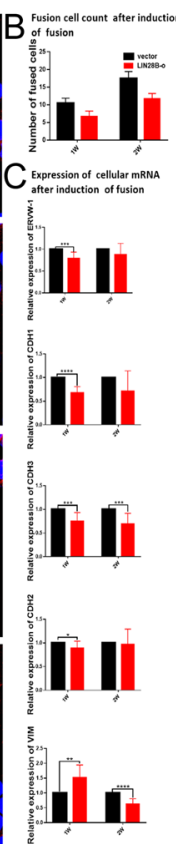

Figure 4 LIN28B inhibits cell fusion. BeWo cells were transfected with (vector) and LIN28B overexpression (LIN28B-o) plasmids in the blank vector control group. The fusion was induced by $50 \mu \mathrm{M}$ FSK and $0.1 \%$ DMSO. After 1 or 2 weeks of continuous culture, immunofluorescence staining was carried out, and the corresponding RNA was observed or extracted by confocal microscope for qPCR test. (A) $\beta$-Catein labeled cell membrane (red, DAPI labeled nucleus (blue). (B) The number of fused cells ( $\geq 3$ nuclear fusions) counted under confocal microscopy after induction of fusion. (C) qPCR was used to detect the mRNA expression levels of BeWo cell fusion-associated factors ERVW-1, CDH1, CDH3, CDH2, and VIM. The number of experimental replicates and the number of replicates within each experiment were both three times. $* * \star \star P<0.0001$, $\star \star \star P<0.001, * * P<0.01, * P<0.05$. development, skeletal muscle and bone development, and immune defense responses (Pötgens et al. 2004, Deng et al. 2017). During the development of the human placenta, it undergoes primary synthesis and secondary integration. When cytotrophoblast cells are fused to the syncytiotrophoblast, they are regulated by various cytokines and growth factors (Maltepe et al. 2010, Gamage et al. 2016, Turco et al. 2018). Syncytin-1 (ERVW-1) was the first molecule to be ascribed a direct ability to promote cell fusion (Pötgens et al. 2004). Canfield et al. (2019) demonstrated that LIN28B plays a role in preeclampsia by reducing syncytialization and that JEG3-knockout of LIN28B in cells significantly decreased SYN-1 while LIN28B overexpression in HTR8/SVneo cells decreased TNF- $\alpha$ expression. Hypoxic culture significantly decreased the expression of LIN28B and SYN-1 in BeWo and EG3 cells and increased the expression of TNF- $\alpha$. EMT is a biological process in which differentiated epithelial cells lose epithelial characteristics and acquire mesenchymal migration. This phenomenon not only plays an important role in tumor invasion and migration, formation of endoderm, and primitive intestinal lumen but also participates in cellfusion processes (Asli \& Harvey 2013, Pei et al. 2019). Lu et al. (2016) speculated that EMT may play a role in the trophoblast cell assembly process and that Twist 1 promotes human placental tissue. Seabrook et al. (2013) showed that knockdown of LIN28A in human trophoblast-like ACH-3P cell lines induced spontaneous syncytialization in the early pregnancy. In the present study, we found that after overexpression of LIN28B the expression level of ERVW-1 decreased, the expression of E-cadherin and P-cadherin increased and that the expression of the interstitial-related factors vimentin and $\mathrm{N}$-cadherin fluctuated greatly. The number of cells fused was also lower than in the control group, and cell-fusion ability was attenuated. Our results indicated that by overexpression of LIN28B and induction of fusion, cell epithelial characteristics increased, while mesenchymal cells fluctuated. This may be because EMT is a transient and reversible process, consistent with studies by Li et al. (2017) where the EMT expression at different timepoints during hESC differentiation was at a dynamic level. Generally, secondary syncytium exerts obvious effects after 12 weeks of gestation, when the synthesized contracted trophoblast cells play an important role in providing nutrition and gas exchange. The invasive capability of EVT manifests a significant time limit that only occurs in the early pregnancy (Burrows et al. 1996, Chakraborty et al. 2002). Overexposure of cytotrophoblast cells in the early pregnancy, then, will result in the differentiation of invasive EVT cells, with a concomitant diminution in invasive ability accompanied by implantation failure and, ultimately, early abortion.

Several studies have shown that LIN28 plays a role in regulating stem cell activity, including self-renewal and differentiation. Therefore, during the early stages of embryonic development, early gene expression in the placenta is high, and cells continuously develop and differentiate (Park et al. 2017, West et al. 2019). LIN28 is also a key factor in the regulation of developmental differentiation. In the present study, we determined 
the expression of LIN28B in villous tissue from some patients with early URSA due to the inherent cellular capabilities for apoptosis, invasion, migration, and cell fusion. The cells in vivo exert their functions through various coordination mechanisms. This study has a certain limitation and cannot directly prove whether lin28b plays the same role in vivo as in vitro cells, but we will conduct further studies to prove the role of LIN28B in vivo cells or in URSA animal models. In the later stage, the peripheral blood expression of LIN28B-related miRNA Let-7 may be detected to predict or interfere with the occurrence of miscarriage. Our future aims are to further confirm that LIN28B is a contributing factor in early abortion diseases and participates in potential mechanisms underlying placental differentiation, development, and function, thus providing a foundation for further molecular research in this area.

\section{Conclusions}

The expression of LIN28B is decreased with URSA, inhibiting cell invasion, and migration, and promoting apoptosis and fusion. Dystrophic dysfunction, then, may be one of the causes of miscarriage.

\section{Declaration of interest}

The authors declare that there is no conflict of interest that could be perceived as prejudicing the impartiality of the research reported.

\section{Funding}

This experiment was supported by funding from the National Nature Science Foundation of China (81300484); the Natural Science Foundation of Guangdong Province, China (2018A0303130308). The manuscript editing and publishing fees of this paper will be funded by the Guangdong provincial medical research fund (B2019007) and the Zhanjiang City Financial Fund Technology Competitive Fund (2019A01023).

\section{Ethics approval and consent to participate}

The protocol governing the subjects of this study was approved by the Ethics Committee of the Affiliated Hospital of Guangdong Medical University (PJ2013014). All of the patients signed informed consent forms.

\section{Availability of data and materials}

All data generated or analyzed during this study are included in this article.

\section{Author contribution statement}

Q Y H: implementation of overall research, data analysis, and draft writing. Y R N and CX W: complete immunohistochemistry and cell culture. L J S and $\mathrm{J} Z \mathrm{H}$ : collection of embryonic villi in aborted patients. T Z M: completed manuscript review, revised paper submission. All authors read and approved the final manuscript.

\section{Acknowledgements}

The authors thank LetPub (www.letpub.com) for its linguistic assistance during the preparation of this manuscript. The authors also thank Bing Wei, Xiaoqun Che, and Lin Zou for their help in case collection. The authors are grateful to Meiwen Huang, Yunqing Li, and Cailing Peng for their help in the collection of aborted villi. The authors extend thanks to Lingli Zhang for their help and dedication in the lab.

\section{References}

Ambros V \& Horvitz HR 1984 Heterochronic mutants of the nematode caenorhabditis elegans. Science 226 409-416. (https://doi.org/10.1126/ science.6494891)

Asli NS \& Harvey RP 2013 Epithelial to mesenchymal transition as a portal to stem cell characters embedded in gene networks. BioEssays 35 191-200. (https://doi.org/10.1002/bies.201200089)

Bao SH, Shuai W, Tong J, Wang L, Chen P \& Duan T 2013 Increased Dickkopf-1 expression in patients with unexplained recurrent spontaneous miscarriage. Clinical and Experimental Immunology 172 437-443. (https://doi.org/10.1111/cei.12066)

Burrows TD, King A \& Loke YW 1996 Trophoblast migration during human placental implantation. Human Reproduction Update 2 307-321. (https://doi.org/10.1093/humupd/2.4.307)

Canfield J, Arlier S, Mong EF, Lockhart J, Vanwye J, GuzelogluKayisli O, Schatz F, Magness RR, Lockwood CJ, Tsibris JCM, et al. 2019 Decreased LIN28B in preeclampsia impairs human trophoblast differentiation and migration. FASEB Journal 33 2759-2769. (https://doi.org/10.1096/fj.201801163R)

Chakraborty C, Gleeson LM, Mckinnon T \& Lala PK 2002 Regulation of human trophoblast migration and invasiveness. Canadian Journal of Physiology and Pharmacology 80 116-124. (https:// doi.org/10.1139/y02-016)

Chan HW, Lappas M, Yee SW, Vaswani K, Mitchell MD \& Rice GE 2013 The expression of the let-7 miRNAs and Lin28 signalling pathway in human term gestational tissues. Placenta 34 443-448. (https://doi. org/10.1016/j.placenta.2013.02.008)

Chang CW, Wakeland AK \& Parast MM 2018 Trophoblast lineage specification, differentiation and their regulation by oxygen tension. Journal of Endocrinology 236 R43-R56. (https://doi.org/10.1530/JOE17-0402)

Coomarasamy A, Williams H, Truchanowicz E, Seed PT, Small R, Quenby S, Gupta P, Dawood F, Koot YE, Bender Atik R, et al. 2015 A randomized trial of progesterone in women with recurrent miscarriages. New England Journal of Medicine 373 2141-2148. (https:// doi.org/10.1056/NEJMoa1504927)

Davis AP, Benninghoff AD, Thomas AJ, Sessions BR \& White KL 2015 DNA methylation of the LIN28 pseudogene family. BMC Genomics 16 287. (https://doi.org/10.1186/s12864-015-1487-3)

Deng S, Azevedo M \& Baylies M 2017 Acting on identity: myoblast fusion and the formation of the syncytial muscle fiber. Seminars in Cell and Developmental Biology 72 45-55. (https://doi.org/10.1016/j. semcdb.2017.10.033)

Gamage TK, Chamley LW \& James JL 2016 Stem cell insights into human trophoblast lineage differentiation. Human Reproduction Update 23 77-103. (https://doi.org/10.1093/humupd/dmw026)

Huang Q, Chen H, Wang F, Brost BC, Li J, Gao Y, Li Z, Gao Y \& Jiang SW 2014 Reduced syncytin-1 expression in choriocarcinoma BeWo cells activates the calpain1-AIF-mediated apoptosis: implication for preeclampsia. Cellular and Molecular Life Sciences 71 3151-3164. (https://doi.org/10.1007/s00018-013-1533-8)

Huppertz B 2008 The anatomy of the normal placenta. Journal of Clinical Pathology 61 1296-1302. (https://doi.org/10.1136/jcp.2008.055277)

Knöfler M \& Pollheimer J 2013 Human placental trophoblast invasion and differentiation: a particular focus on wnt signaling. Frontiers in Genetics 4 190. (https://doi.org/10.3389/fgene.2013.00190)

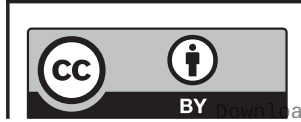

This work is licensed under a Creative Commons Attribution 4.0 International License. 
Li Q, Hutchins AP, Chen Y, Li S, Shan Y, Liao B, Zheng D, Shi X Li Y, Chan WY, et al. 2017 A sequential EMT-MET mechanism drives the differentiation of human embryonic stem cells towards hepatocytes. Nature Communications 8 15166. (https://doi.org/10.1038/ ncomms15166)

Lin X, Shen J, Dan Peng, He X, Xu C, Chen X, Tanyi JL, Montone K, Fan Y, Huang Q, et al. 2018 RNA-binding protein LIN28B inhibits apoptosis through regulation of the AKT2/FOXO3A/BIM axis in ovarian cancer cells. Signal Transduction and Targeted Therapy 323. (https://doi.org/10.1038/s41392-018-0026-5)

Lozoya T, Domínguez F, Romero-Ruiz A, Steffani L, Martínez S, Monterde M, Ferri B, Núñez MJ, AinhoaRomero-Espinós, Zamora O, et al. 2014 The Lin28/Let-7 system in early human embryonic tissue and ectopic pregnancy. PLOS ONE 9 e87698. (https:// doi.org/10.1371/journal.pone.0087698)

Lu X, He Y, Zhu C, Wang H, Chen S \& Lin HY 2016 Twist1 is involved in trophoblast syncytialization by regulating GCM1. Placenta 39 45-54. (https://doi.org/10.1016/j.placenta.2016.01.008)

Maltepe E, Bakardjiev AI \& Fisher SJ 2010 The placenta: transcriptional, epigenetic, and physiological integration during development. Journal of Clinical Investigation 120 1016-1025. (https:// doi.org/10.1172/JCI41211)

Mekinian A, Loire-Berson P, Nicaise-Roland P, Lachassinne E, Stirnemann J, Boffa MC, Chollet-Martin S, Carbillon L \& Fain 02012 Outcomes and treatment of obstetrical antiphospholipid syndrome in women with low antiphospholipid antibody levels. Journal of Reproductive Immunology 94 222-226. (https://doi. org/10.1016/j.jri.2012.02.004)

Monk D 2015 Genomic imprinting in the human placenta. American Journal of Obstetrics and Gynecology 213 (Supplement) S152-S162. (https://doi.org/10.1016/j.ajog.2015.06.032)

Moore T 2001 Genetic conflict, genomic imprinting and establishment of the epigenotype in relation to growth. Reproduction 122 185-193. (https://doi.org/10.1530/rep.0.1220185)

Motedayyen H, Zarnani AH, Tajik N, Ghotloo S \& Rezaei A 2018 Immunomodulatory effects of human amniotic epithelial cells on naive CD4(+) T cells from women with unexplained recurrent spontaneous abortion. Placenta 71 31-40. (https://doi.org/10.1016/j. placenta.2018.06.008)

Ogasawara M, Aoki K, Okada S \& Suzumori K 2000 Embryonic karyotype of abortuses in relation to the number of previous miscarriages. Fertility and Sterility 73 300-304. (https://doi.org/10.1016/ S0015-0282(99)00495-1)

Park JH, Park BW, Kang YH, Byun SH, Hwang SC, Kim DR, Woo DK \& Byun JH 2017 Lin28a enhances in vitro osteoblastic differentiation of human periosteum-derived cells. Cell Biochemistry and Function 35 497-509. (https://doi.org/10.1002/cbf.3305)

Pei D, Shu X, Gassama-Diagne A \& Thiery JP 2019 Mesenchymalepithelial transition in development and reprogramming. Nature Cell Biology 21 44-53. (https://doi.org/10.1038/s41556-018-0195-z)

Pollheimer J, Vondra S, Baltayeva J, Beristain AG \& Knöfler M 2018 Regulation of placental extravillous trophoblasts by the maternal uterine environment. Frontiers in Immunology 9 2597. (https://doi. org/10.3389/fimmu.2018.02597)

Pötgens AJ, Drewlo S, Kokozidou M \& Kaufmann P 2004 Syncytin: the major regulator of trophoblast fusion? Recent developments and hypotheses on its action. Human Reproduction Update 10 487-496. (https://doi.org/10.1093/humupd/dmh039)

Recurrent Spontaneous Abortion 2012 Evaluation and treatment of recurrent pregnancy loss: a committee opinion. Fertility and Sterility $\mathbf{9 8}$ 1103-1111. (https://doi.org/10.1016/j.fertnstert.2012.06.048)

Seabrook JL, Cantlon JD, Cooney AJ, Mcwhorter EE, Fromme BA, Bouma GJ, Anthony RV \& Winger QA 2013 Role of LIN28A in mouse and human trophoblast cell differentiation.
Biology of Reproduction 89 95. (https://doi.org/10.1095/ biolreprod.113.109868)

Shyh-Chang N \& Daley GQ 2013 Lin28: primal regulator of growth and metabolism in stem cells. Cell Stem Cell 12 395-406. (https://doi. org/10.1016/j.stem.2013.03.005)

Stephenson MD, Awartani KA \& Robinson WP 2002 Cytogenetic analysis of miscarriages from couples with recurrent miscarriage: a case-control study. Human Reproduction 17 446-451. (https://doi. org/10.1093/humrep/17.2.446)

Thornton JE \& Gregory RI 2012 How does Lin28 let-7 control development and disease? Trends in Cell Biology 22 474-482. (https:// doi.org/10.1016/j.tcb.2012.06.001)

Turco MY, Gardner L, Kay RG, Hamilton RS, Prater M, Hollinshead MS, Mcwhinnie A, Esposito L, Fernando R, Skelton H, et al. 2018 Trophoblast organoids as a model for maternal-fetal interactions during human placentation. Nature 564 263-267. (https://doi.org/10.1038/s41586-018-0753-3)

Velicky P, Knöfler M \& Pollheimer J 2016 Function and control of human invasive trophoblast subtypes: intrinsic vs. maternal control. Cell Adhesion and Migration 10 154-162. (https://doi.org/10.1080/1933 6918.2015.1089376)

West RC, Mcwhorter ES, Ali A, Goetzman LN, Russ JE, GonzalezBerrios CL, Anthony RV, Bouma GJ \& Winger QA 2019 HMGA2 is regulated by LIN28 and BRCA1 in human placental cells. Biology of Reproduction 100 227-238. (https://doi.org/10.1093/biolre/ioy183)

Windsperger K, Dekan S, Pils S, Golletz C, Kunihs V, Fiala C Kristiansen G, Knöfler M \& Pollheimer J 2017 Extravillous trophoblast invasion of venous as well as lymphatic vessels is altered in idiopathic, recurrent, spontaneous abortions. Human Reproduction 32 1208-1217. (https://doi.org/10.1093/humrep/dex058)

Wu F, Tian F, Zeng W, Liu X, Fan J, Lin Y \& Zhang Y 2017 Role of peroxiredoxin2 downregulation in recurrent miscarriage through regulation of trophoblast proliferation and apoptosis. Cell Death and Disease 8 e2908. (https://doi.org/10.1038/cddis.2017.301)

Xu Z, Hou X, Lv H, Sun B, Cui Y, Liu L \& Rong F 2017 Expression of liver receptor homolog-1 (LRH-1) in villi and decidua of patients with unexplained recurrent spontaneous abortion. Medical Science Monitor 23 2445-2452. (https://doi.org/10.12659/msm.904645)

Xu Y, Ban Y, Ran L, Yu Y, Zhai S, Sun Z, Zhang J, Zhang M, Hong T, Liu R, et al. 2019 Relationship between unexplained recurrent pregnancy loss and 5,10-methylenetetrahydrofolate reductase) polymorphisms. Fertility and Sterility 111 597-603. (https://doi. org/10.1016/j.fertnstert.2018.11.011)

Yang C, Lim W, Bazer FW \& Song G 2018 Homosalate aggravates the invasion of human trophoblast cells as well as regulates intracellular signaling pathways including PI3K/AKT and MAPK pathways. Environmental Pollution 243 1263-1273. (https://doi.org/10.1016/j. envpol.2018.09.092)

Zhao G, Yang C, Yang J, Liu P, Jiang K, Shaukat A, Wu H \& Deng G 2018 Placental exosome-mediated Bta-miR-499-Lin28B/let-7 axis regulates inflammatory bias during early pregnancy. Cell Death and Disease 9 704. (https://doi.org/10.1038/s41419-018-0713-8)

Zhou Y, Fisher SJ, Janatpour M, Genbacev O, Dejana E Wheelock M \& Damsky CH 1997 Human cytotrophoblasts adopt a vascular phenotype as they differentiate: a strategy for successful endovascular invasion? Journal of Clinical Investigation 99 2139-2151. (https://doi.org/10.1172/JCI119387)

Received in final form 14 July 2021

Accepted 12 August 2021

Accepted Manuscript published online 16 August 2021

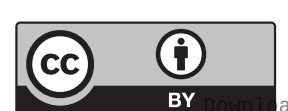

This work is licensed under a Creative Commons Attribution 4.0 International License. 